2018-12-24

\title{
Architecture for a Social Assistive Robot in Cardiac Rehabilitation
}

Casas, J

http://hdl.handle.net/10026.1/13637

10.1109/CCRA.2018.8588133

2018 IEEE 2nd Colombian Conference on Robotics and Automation, CCRA 2018

All content in PEARL is protected by copyright law. Author manuscripts are made available in accordance with publisher policies. Please cite only the published version using the details provided on the item record or document. In the absence of an open licence (e.g. Creative Commons), permissions for further reuse of content should be sought from the publisher or author. 
This is the author's manuscript that was accepted on October 2, 2018. The final version of this work is published by IEEE in 2018 IEEE 2nd Colombian Conference on Robotics and Automation (CCRA), available at DOI: 10.1109/CCRA.2018.8588133. This work is made available online in accordance with the publisher's policies. Please refer to any applicable terms of use of the publisher. 


\title{
Architecture for a Social Assistive Robot in Cardiac Rehabilitation
}

\author{
Jonathan Casas ${ }^{1}$, Nathalia Céspedes Gomez ${ }^{1}$, Emmanuel Senft ${ }^{2}$, Bahar Irfan $^{2}$, Luisa F. Gutiérrez ${ }^{3}$ \\ Mónica Rincón ${ }^{3}$, Marcela Múnera ${ }^{1}$, Tony Belpaeme ${ }^{4,2}$, Carlos A. Cifuentes ${ }^{1}$
}

\begin{abstract}
Social robots are demonstrating to have potential in several health care applications, especially in rehabilitation areas. This paper presents an architecture for a socially assistive robot system for cardiac rehabilitation, based on a model-controller structure through a finite-state machine and a behaviour module. The platform has been designed to provide social support and assistance during the therapy, aiming to improve the quality of the provided service, as well as the engagement and performance of the patients. This architecture has been tested under clinical conditions with a patient during a typical therapy session. The results show that a suitable performance to the different situations presented during the session and providing a robust framework to further develop the robot's behavior towards a more natural and intuitive interaction with patients.
\end{abstract}

\section{INTRODUCTION}

Cardiovascular diseases (CVDs) are disorders of the heart and blood vessels and include cerebrovascular disease, rheumatic heart disease and other conditions [1]. CVDs are the cause of 17.7 million deaths every year, approximately $31 \%$ of the world population [1]. As part of the solution to CVDs, Cardiac Rehabilitation (CR) appears as an important strategy that seeks to improve the quality of life of patients who suffered a heart disease. These strategies include exercise regimens and conventional procedures that can be improved using technologies that support different aspects of the therapy such as measurements, physical and cognitive assistance, evaluation processes, data analysis among others.

In this context, there are different technologies that have been implemented in similar applications aiming to enhance medical services. Augmented Reality (AR) and Virtual Reality (VR) for instance, are a new technological systems based on interactive experience that allows perceive virtual environments in the real world [2]. For stroke rehabilitation, Merians et al implemented a virtual reality system using 3D graphics, showing improvement in different motor tasks

This work was supported in part by the Industry Academia Partnership Programme Colombia UK (IAPP/1516/137 / 2016-2018), HumanRobot Interaction Strategies for Rehabilitation based on Socially Assistive Robotics in collaboration with Plymouth University, the EU H2020 Marie Sklodowska-Curie Actions ITN project APRIL (grant 674868), the EU FP7 project DREAM (grant 611391) and Colciencias SORCAR project (grant 813-2017) in collaboration with Fundacion Cardioinfantil-Instituto De Cardiologia.

${ }^{1}$ Center for Biomechatronics, Colombian School of Engineering Julio Garavito, Bogotá, Colombia name. surnamedescuelaing.edu.co

${ }^{2}$ Centre for Robotics and Neural Systems (CRNS), University of Plymouth, Plymouth, UK name. surnamedplymouth.ac.uk

${ }^{3}$ Fundación Cardioinfantil - Instituto de Cardiología, Bogotá, Colombia

${ }^{4}$ IDLab - imec, Ghent University, Ghent, Belgium
[3]. Luo et al designed an augmented reality rehab system for post-stroke hand rehabilitation, the system was evaluated on pilot case studies during 6-week training, showing user acceptance of the technology and potential for beneficial effects [4]. Furthermore, Jack et al proposed an augmented reality framework for post-stroke rehabilitation to perform repetitive motor tasks during therapy, obtaining an increasing of patient's encouragement [5]. Although AR applications have shown promising outcomes in different assistive environments, they are mostly focused on improving the physical capabilities of the patient. To provide a comprehensive assistive tool, technologies such as Virtual Companions have emerged, aiming to approach not only physical skills but also cognitive and social aspects during therapy. Virtual companions have been explored for cardiac patients in different scenarios, mostly stroke rehabilitation.

Bickmore and Picard [6] presented a virtual relational agent for exercise adoption. The agent has an animated human body and interacts with patients in simulated conversations. The most important result was a reported desire by the patients to continue working on terms of improving the adherence. In stroke rehabilitation, there are assorted research studies; Sakamoto et al designed a stroke-based interface for home therapies with a vaccuming robot. The architecture allows users to control robots intuitively and also perform upper-limb rehabilitation through training gestures; as a results of preliminary study shows a potential tool for home rehabilitation [7]. Groos et al, describes a robotic rehabilitation assistance for proprioceptive gait exercises in self-training during therapies (ROREAS project), the robot could fulfill the expectations regarding technical tasks, on the other hand as a coach robot the agent proved to be reliable and suitable [8]

Most recently, another approach that has demonstrated promising results in education and rehabilitation is the Socially Assistive Robotics (SAR), which is also focused on providing assistance trough social interactions [9], unlike virtual agents (VA), social robots present a physical embodiment. A robot's body is tangible while virtual agent's body is digitally generated using computer algorithms [10]. This approach has been also explored for cardiac patients in post-stroke rehabilitation [11], where a social robot provided monitoring and encouragement during therapy. Moreover in [12], SAR is evaluated for cardiac rehabilitation with "CLARA", a hands-off physical therapy assistant. CLARA aims to reducing the effects of nursing shortage, improve the rehabilitation processes by helping patients during 
repetitive spirometry exercises, some of skills of the robot were navigation in medical environments, social interaction and exercise tracking. The study shows that CLARA's functionalities were performed successfully, and patients were more motivated.

In previous work [13], we presented and validated a Human-Robot sensor interface under laboratory conditions with healthy patients; physiological parameters as: heart rate and gait spatio-temporal patterns and cognitive parameters as: perception on exertion during the exercise were measured. Moreover, Casas et al extended this work to the clinic, aiming to asses the system under clinical conditions with real patients [14]; where a patient performed therapy with the robot companion, showing a promising potential of the application. However, a more reliable framework is needed to assure robustness of the system in the long term. In this regard, this work presents a social robot architecture using a NAO robot, based on a model-controller approach, aiming to build upon previous work presented and offer a suitable platform to the system.

The remainder of this paper is organized as follows. Section II describes cardiac rehabilitation conventional therapy procedures. Section III introduces the proposed architecture design, a briefly description of the software architecture and robot approach, followed by Section IV which presents the results of the SAR design. Finally conclusions and future work proposed for this research is presented.

\section{REHABilitation CONTEXT}

CR combines prescriptive exercise training with coronary risk factor modification in patients with established heart disease [15]. The main goals of CR are to achieve a full recovery in terms of reach optimal physical, mental and social status and modify coronary risk factor to reduce subsequent mortality due to cardiovascular illness [16]. CR is focused on two aspects: (1) physiological aspects focused on the physiological performance of the patients such as cardiovascular functioning, aerobic capabilities during exercise etc, and (2) cognitive aspects related to the cognition processes that involves the language, perception, motivation, attention and memory [17]. These aspects are essential to evaluate the patient throughout the rehabilitation time and also measure their long-term performance.

Given that Cardiac Rehabilitation is an exercise-based program, the therapies consist of aerobic and anaerobic training. Prescribed exercise for patients with coronary heart disease is based on individualized regimens [18]. Walking and using bicycle ergometer devices have been the preferred exercise (See Figure 1), the number of sessions are typically 36 depending on the phase (outpatient/phase II and maintenance program/phase III) and the exercise intensity changes according patients level of exertion. The major challenge of the assistive technologies is to increase patient's engagement and to provide monitoring, as well as encouragement during these physical activities tacking into account the increasing dropout rates. Despite the evidence of the benefits of (CR), about one third of patients participate in the programs [19]. For example, US and Australia figures suggest that only $15 \%-30 \%$ of patients participate in such programs. Some factors that can act as an adherence barrier include belief variables, age, annual income, level of education, cardiac functional status, mood state and social support [20], [21].

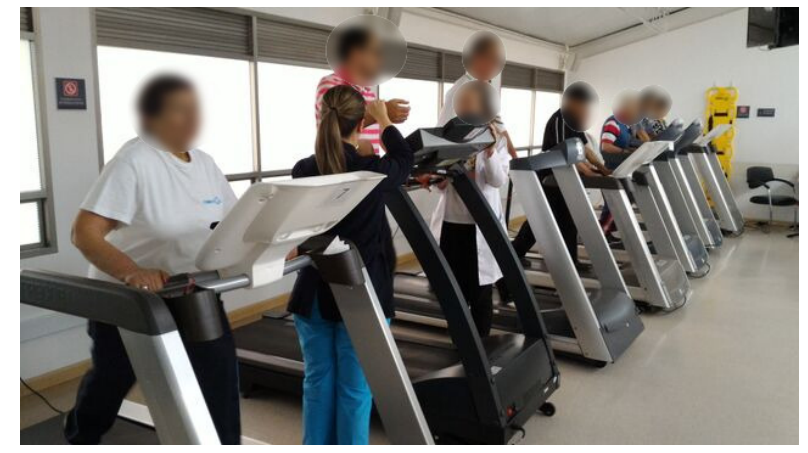

Fig. 1. Conventional scenario for Cardiac Rehabilitation.

Under the previous conditions, the purpose of this work is to develop a modular robot architecture to provide a platform that integrates a social robot into the CR therapy. This architecture is aiming to allow the development of an autonomous robot with custom behaviours that responds properly to the context of the therapy. Next section describes the proposed architecture, incorporating a social robotic platform NAO in the system.

\section{ArChitecture Design}

In general, the interface is conformed by 4 main modules: (1) Graphical User Interface (GUI) which allows the user interaction with the system, (2) Sensor Manager dedicated to handle all sensory data acquisition and processing, (3) Storage System that allows communication and data transmission between modules and (4) Social Robot to provide social interaction during the therapy.

According to the context in which the SAR system is deployed, this architecture is designed to be integrated as an additional module to the sensor interface presented in ([13]). The architecture presented here is meant to be integrated to the Social Robot module. Figure 2. shows the main architecture conformed by three layers, namely Application layer, Model-Controller Layer and Hardware Layer. These layers are described below.

\section{A. Application Layer}

This layer describes the connectivity with the external application, in this case for the Human-Robot sensor interface (See. [13]). The application runs on a PC or tablet and creates the Social Assistive Robot Interface (SARI) which receives sensory data acquired by the sensor interface and events associated to the system that feed the Finite-State Machine (FSM) In the next layer. 


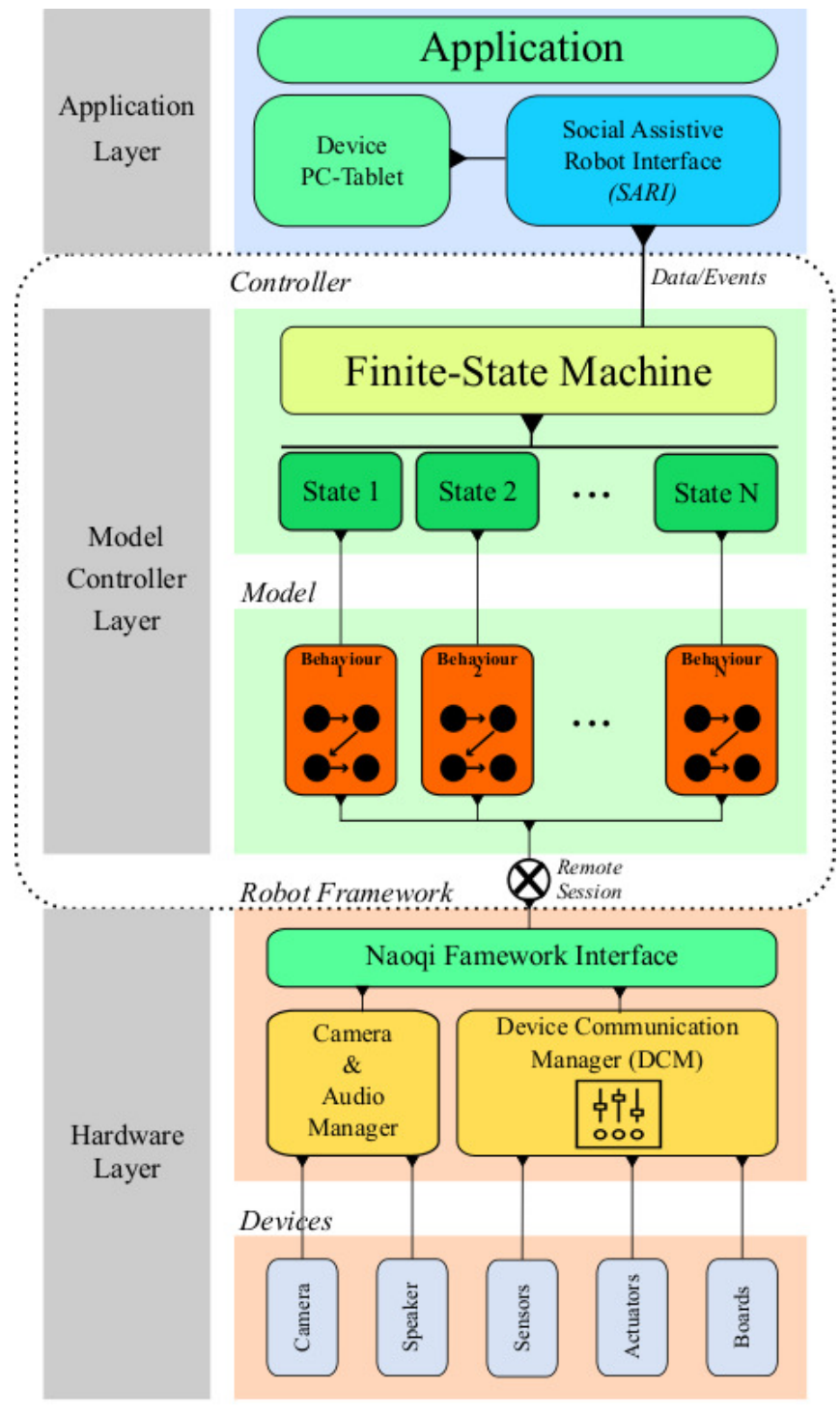

Fig. 2. Three-layers architecture for Social Robot Model platform at Institute of Cardio-Infantil.

\section{B. Model-Controller Layer}

The proposed work presented in this article is composed of two main modules: the Robot Controller and the Robot Model. Together, they provide the decision framework that enables the robot to interact, with a determined behaviour, with the user and the system according to the data obtained from the Application Layer, sent trough the SARI.

1) Robot Controller: This module defines the robot's state in a given situation. As described in the application layer, sensory data is sent to the controller and received by the FSM. The FSM evaluates the current state, as well as the data received and determines the next state of the system. Once the new state is calculated, the information is sent to the Robot Model to adopt the corresponding behaviour. The description of the FSM for a social robot in the CR therapy is described below.

Aiming to understand the context of the therapy, previ- ous observations were performed at the Fundación Cardio Infantil-Instituto de Cardiología (FCI-IC). From these observations it was possible to understand how a conventional session is developed and how the interaction Patient-Therapist is established. Three main functions performed by the health professional were identified: motivation, monitoring and assistance that were considered to design a suitable FSM for the therapy.

As mentioned above, the robot tasks are classified in three types: (1) Motivation provided periodically (tipically each 5 minutes) through encouraging speech and movement interventions of the robot, (2) Monitoring which is the continuous analysis of the data (each second). This is considered the main tasks carried out by the robot, since most of the time it remains in this state (See Figure 3.). (3) Assistance is meant to be provided when warning or emergency situations are perceived.

Figure 3. shows the configuration of the FSM for cardiac therapy. As depicted in the graph, the initial state (called start) initializes the FSM and triggers the Welcome state. This state performs a simple intervention to welcome the user to the system and start the therapy. Once the welcome state finishes, it activates the monitoring state that receives sensory data at a frequency of $1 \mathrm{~Hz}$. As observed in Figure 3 , the monitoring state is located as the central state, since all the data analysis is carried out there. According to this analysis it takes the decision to remain in the current state or to trigger another one such as motivation, posture correction ${ }^{1}$ and Borg Scale ${ }^{2}$. However, after one of these events finishes, they return to the monitor state.

Additionally, the Warning is triggered when the data provided is reaching critical values regarding the patient's physical profile. During this state a validation of these conditions is performed, hence according to the result the system can return to the monitoring state and continue the therapy or trigger the Emergency state that requests the health professionals to provide assistance. The Emergency state can be triggered as mentioned before, or it can be directly activated from the monitoring state if the data analysis provides critical results, indicating that immediate assistance is required. This state can also return to the monitoring state when health professionals have controlled the situation and the patient is able to continue or in the other case, when the situation is critical, the system must activate the shutdown state and finish the session

Each state defines a behaviour that is associated to each situation. Once the next state is defined, the Controller communicates with the Model sublayer to adopt the desired behaviour. This layer is described in the next section.

2) Robot Behavior: As described above, this sublayer receives the information from each state to activate a de-

\footnotetext{
${ }^{1}$ Posture correction occurs when the patient tilted the head down, keeping the head at that position could cause dizziness and it is consider a risk factor during the therapy

${ }^{2}$ The Borg Scale is considered a qualitative exertion rate that is used to asses the intensity of the activity and how exhausted the patient is perceiving. This values is asked periodically by the system
} 


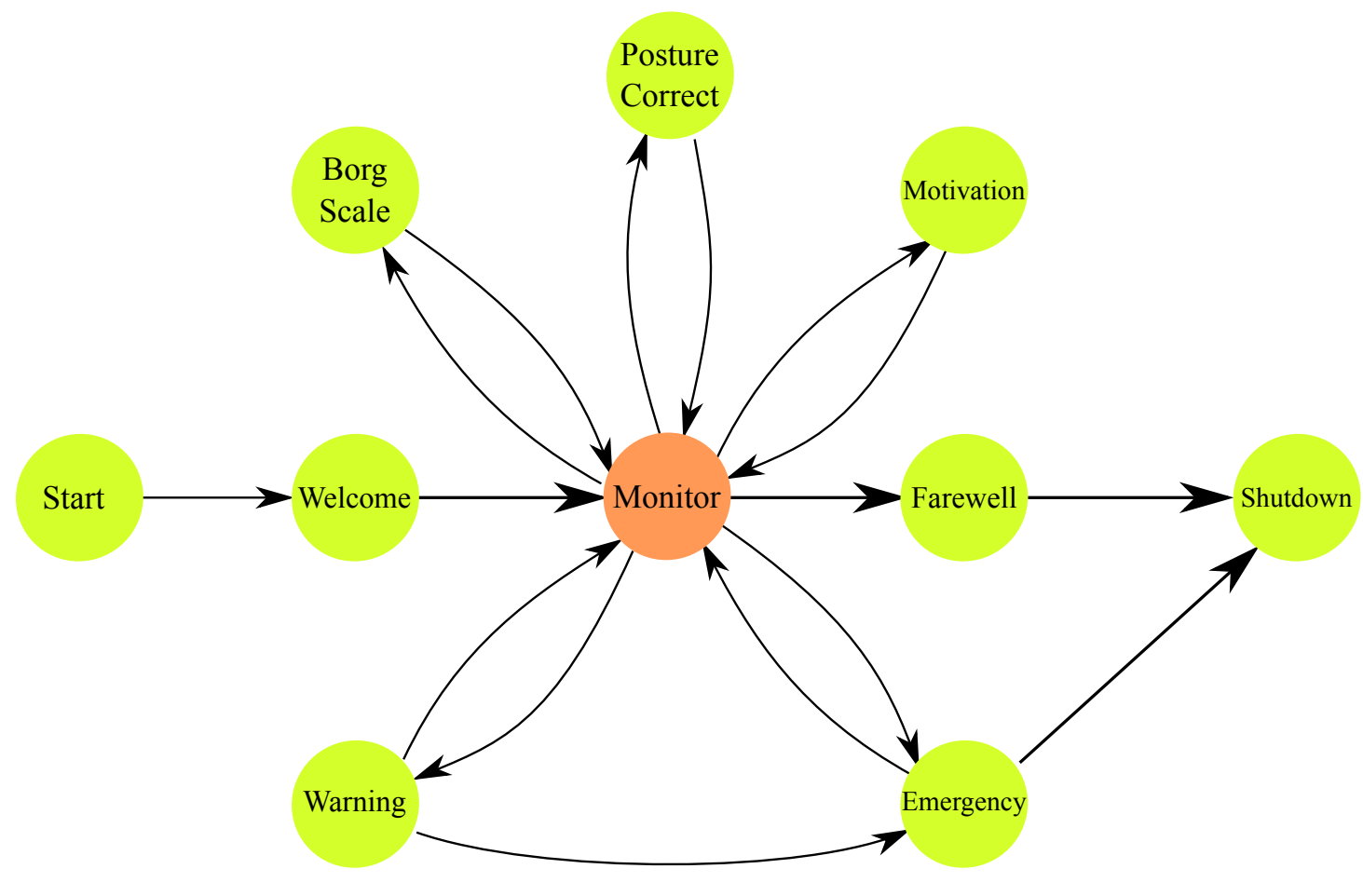

Fig. 3. Robot behaviors Finite-State Machine (FSM).

termined behavior (See Figure 2.). A behavior is considered as a sequence of actions that the robot must perform. Each sequence requires some specific resources, namely cameras, speakers, speech synthesizers and other resources from the robot. Therefore, a behaviour can be structured as a time-line that allocates resources in different instants of time.

For this application, most of the behaviours that were implemented, use similar resources such as speech synthesizer and animations. Since the objective of the social robot is to assist therapies through social interaction, the dialog and body language are more relevant. However, the emergency state requires from other resources such as tactile sensors that are activated when the health professionals attend to its assistance requests. In this case, the professional touch the sensor to stop the emergency behaviour and indicates to return to the monitoring state or to stop the therapy. Representation of a behaviour structure is illustrated in Figure 4. Where different resources (Speech, Motion, Leds) are located in specific times to accomplish the desired behaviour.

Until this point, all the process is carried out on the application device, namely a PC or tablet. Once the sequence of the resources is defined, this information is deployed to the robot platform trough a remote session that enables the interface to communicate with the Hardware Layer which contains all hardware resources. Next section describes this layer in more detail.

\section{Platform Specifications \\ D. Hardware Layer}

As mentioned in the previous section, this layer is accessed remotely from the application device. In first

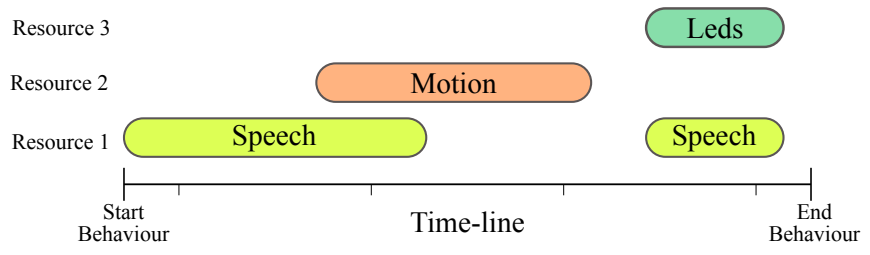

Fig. 4. Conventional structure for NAO robot behavior.

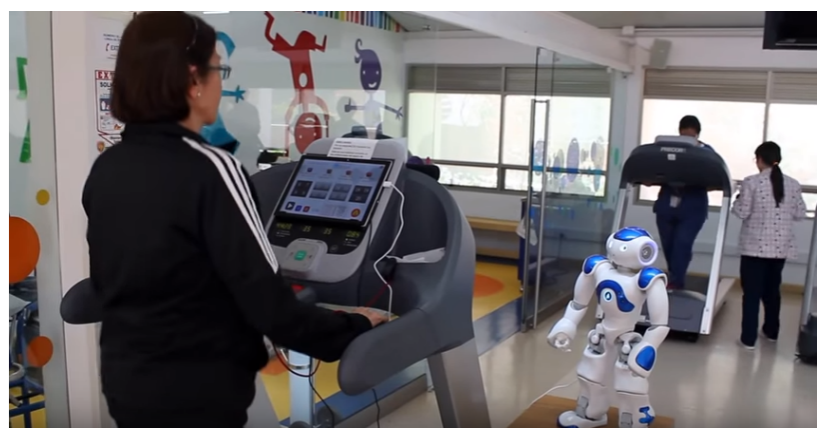

Fig. 5. Experimental setup at Fundación Cardioinfantil-Instituto de cardiología (FCI-IC)

place, there is an Interface, which for this application is the Naoqi Framework ${ }^{3}$, that enables the access to the robot's resources as services via TCP-IP protocol. As depicted in Figure 2, the interface manages two modules, Camera \& Audio Manager that administrates camera, microphones and

\footnotetext{
${ }^{3}$ Naoqi framwork is the API that provide access to Aldebaran robots NAO and PEPPER: http://doc.aldebaran.com/2-1/ref/index.html
} 


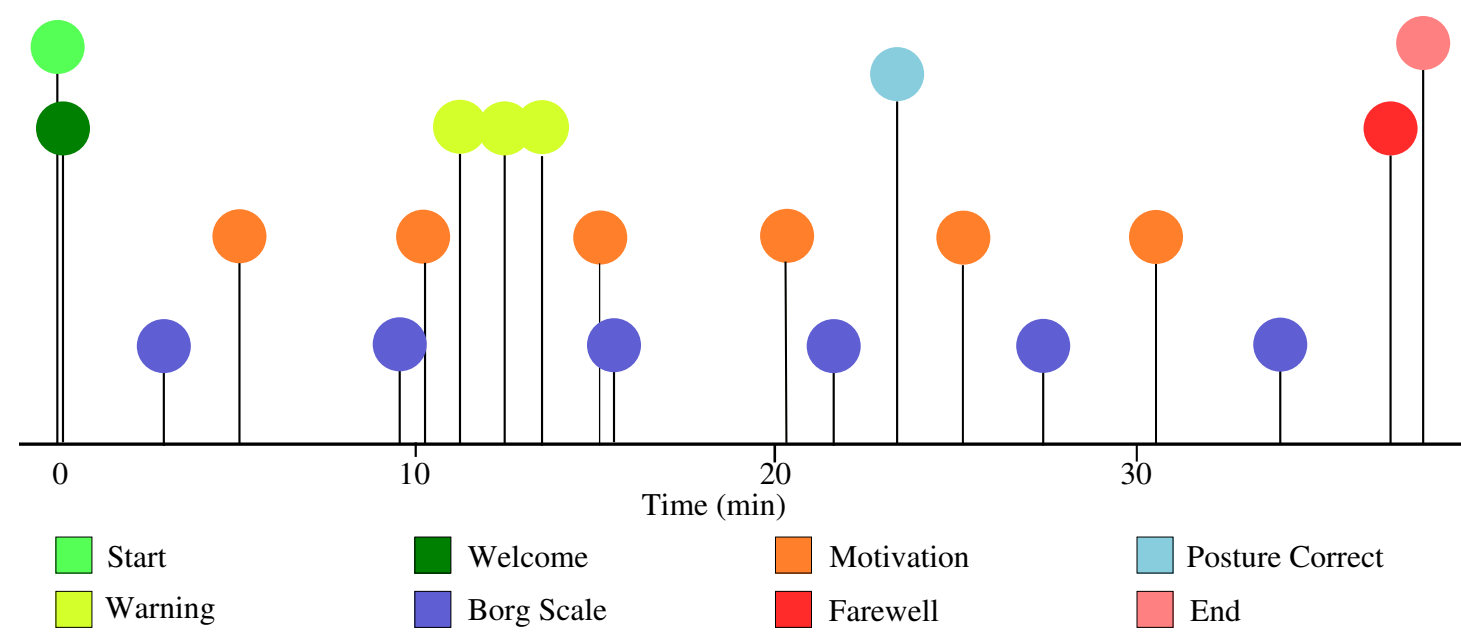

Fig. 6. Event plot of a conventional CR therapy with duration of 38 minutes with the proposed architecture.

speakers. On the other hand, the $D C M^{4}$ module controls the rest of the resources such as sensors, actuators and boards integrated in the robot.

As mentioned before, the robot platform that was integrated in this architecture is the humanoid robot NAO (Aldebaran, France) (see Fig 5). NAO is an autonomous programmable robot with 25 degrees of freedom. The platform includes inertial measurement units to provide stability and space positioning, force-sensing resistors, two bumpers, microphones for sound recognition and sound localization, speakers (for text-to-speech synthesis) and two cameras used in computer vision and recognition applications [22]. Additionally, this robot has a board Atom (Z530 1,6 GHz) and $\mathrm{Wi}$ - $\mathrm{Fi}$ interface IEEE 802,11. Next sections describes the architecture's performance during a real CR therapy.

\section{Performance Assessment}

With the purpose of assessing the architecture's performance, this section describes the results obtained during a conventional CR therapy with a male patient (age: 55 years, height: $1.66 \mathrm{~m}$ and weigh: $75 \mathrm{Kg}$ ) with an acute myocardial infarction. The robot platform is placed on one side of the treadmill (See Figure 5.). As mentioned in the architecture, the interface is remotely connected to the robot and all data and events are transmitted trough this channel.

The architecture was operating for 38 minutes that took the therapy to be carried out. Figure I. shows the occurrences of each event that triggered a specific state of the FSM. The events are classified in colors and the distribution during the the therapy is represented. Table I. summarizes the number of times that the FSM was on each state during the session.

As shown in Table I, states such as welcome, farewell and shutdown have only one occurrence, since they cannot return to its state again. On the other hand, event-depending states and data-depending states were triggered more than

\footnotetext{
${ }^{4}$ See the DCM description in: http://doc.aldebaran.com/21/naoqi/sensors/dcm.html\#dcm
}

TABLE I

NUMBER OF OCCURRENCES OF EACH EVENT DURING A CONVENTIONAL CR THERAPY

\begin{tabular}{lc}
\hline State & Occurrences \\
\hline Welcome & 1 \\
Borg Scale & 6 \\
Motivation & 6 \\
Posture Correct & 1 \\
Warning & 3 \\
Emergency & 0 \\
Farewell & 1 \\
Shutdown & 1 \\
\hline
\end{tabular}

once. This is the case for the motivation state and Borg Scale states, that were triggered both 6 times. However, there was a particular situation for the Posture correct state (triggered only once), since according to the experimenter observations, there were more situations that should have triggered but the system did not respond fast enough.

A similar situation occurred with the warning state that was triggered 3 times, where in all the occasions the patient did not report any emergency. However, the sensor interface detected high parameters and triggered the state repeatedly. During the therapy the emergency state was not triggered as the patient did not presented any complication. As the FSM remains most of the time in the monitor state, it is not taken into account in Table I.

\section{Discussion \& CONCLUSIONS}

With the implementation of the proposed robot architecture, it was possible to integrate a social robot into a $\mathrm{CR}$ therapy, responding accordingly to the different situations presented. The robot was operative during 38 minutes without presenting any technical difficulty (E.g. System failure, disconnection). However, some adjustments to the behavior logic, namely the states sequence of the FSM are required. (1) For the posture correction state it is necessary to increase 
the response time, in order to provide a more accurate correction during the session. As mentioned in the last section, the robot was able to detect only once a bad posture, even when there were more situations that required from this intervention. Additionally, the latency of the response was significantly high since the correction was requested by the robot a few seconds after the patient itself corrected its posture. (2) The warning state requires an inhibition feature. As explained in Section IV, the warning occurred three times and the patient in the first event reported no complications, then after one minute another warning event was triggered, receiving the same response from the patient. Therefore, to avoid a third consecutive repetition and probably more than 3 repetitions, the FSM must verify patients conditions and if these allows to continue, lock the warning state for a determined period and continue with the therapy.

For the future work, it is expected to increase the complexity of the FSM, in order to provide a more natural interaction with the users. As the current system provides the transitions of the states in a deterministic way, the purpose of future contributions is to perform these transitions based on events with a probabilistic distribution of occurrence. Furthermore, the incorporation of learning algorithms (E.g. People recognition, Personalized performance follow-up) is desired in this kind of applications to increase the assistance and support for different patients.

\section{REFERENCES}

[1] WHO (World Health Organization), "World report on disability 2011," American journal of physical medicine rehabilitation Association of Academic Physiatrists, vol. 91, p. 549, 2011.

[2] R. T. Azuma, "A Survey of Augmented Reality," Presence: Teleoperators and Virtual Environments, vol. 6, no. 4, pp. 355-385, aug 1997.

[3] A. S. Merians, D. Jack, R. Boian, M. Tremaine, G. C. Burdea, S. V. Adamovich, M. Recce, and H. Poizner, "Virtual Reality-Augmented Rehabilitation for Patients Following Stroke," Physical Therapy, vol. 82, no. 9, pp. 898-915, sep 2002.

[4] Y. Jia, M. L. Toro, X. Luo, S. Lau, R. Kenyon, and D. Kamper, "Integration of Virtual Reality and an Assistive Device for Hand Rehabilitation Following Stroke," 2007 IEEE/ICME International Conference on Complex Medical Engineering, no. June, pp. 13311336, 2007.

[5] D. Jack, R. Boian, A. Merians, M. Tremaine, G. Burdea, S. Adamovich, M. Recce, and H. Poizner, "Virtual reality-enhanced stroke rehabilitation," IEEE Transactions on Neural Systems and Rehabilitation Engineering, vol. 9, no. 3, pp. 308-318, 2001.

[6] R. W. P. Timothy W. Bickmore, "Establishing and Maintaining LongTerm Human-Computer Relationships," pp. 617-638.

[7] D. Sakamoto, K. Honda, M. Inami, and T. Igarashi, "Sketch and run: a stroke-based interface for home robots," SIGCHI Human Factors in Computing Systems, pp. 197-200, 2009.

[8] H.-M. Gross, K. Debes, E. Einhorn, S. Mueller, A. Scheidig, C. Weinrich, A. Bley, and C. Martin, "Mobile Robotic Rehabilitation Assistant for walking and orientation training of Stroke Patients: A report on work in progress," 2014 IEEE International Conference on Systems, Man, and Cybernetics (SMC), no. SMC, pp. 1880-1887, 2014.

[9] D. Feil-Seifer and M. Mataric, "Socially Assistive Robotics," in 9th International Conference on Rehabilitation Robotics, 2005. ICORR 2005. IEEE, pp. 465-468.

[10] J. Li, "The benefit of being physically present: A survey of experimental works comparing copresent robots, telepresent robots and virtual agents," International Journal of Human Computer Studies, vol. 77, pp. 23-27, 2015. [Online]. Available: http://dx.doi.org/10.1016/j.ijhcs.2015.01.001

[11] M. J. Matarić, J. Eriksson, D. J. Feil-Seifer, and C. J. Winstein, "Socially assistive robotics for post-stroke rehabilitation," Journal of NeuroEngineering and Rehabilitation, vol. 4, no. 1, p. 5, 2007.
[12] Kyong Il Kang, S. Freedman, M. Mataric, M. Cunningham, and B. Lopez, "A Hands-Off Physical Therapy Assistance Robot for Cardiac Patients," 9th International Conference on Rehabilitation Robotics, 2005. ICORR 2005., pp. 337-340.

[13] J. S. Lara, J. Casas, A. Aguirre, M. Munera, M. Rincon-Roncancio, B. Irfan, E. Senft, T. Belpaeme, and C. A. Cifuentes, "Humanrobot sensor interface for cardiac rehabilitation," IEEE International Conference on Rehabilitation Robotics, pp. 1013-1018, 2017.

[14] J. Casas, B. Irfan, E. Senft, L. Gutiérrez, M. Rincon-Roncancio, M. Munera, T. Belpaeme, and C. A. Cifuentes, "Social assistive robot for cardiac rehabilitation: A pilot study with patients with angioplasty," in Companion of the 2018 ACM/IEEE International Conference on Human-Robot Interaction, ser. HRI '18. New York, NY, USA: ACM, 2018, pp. 79-80.

[15] N. F. Gordon and W. L. Haskell, "Comprehensive cardiovascular disease risk reduction in a cardiac rehabilitation setting." The American journal of cardiology, vol. 80, no. 8B, pp. 69H-73H, oct 1997.

[16] "Cardiac rehabilitation programs. A statement for healthcare professionals from the American Heart Association." Circulation, vol. 90, no. 3, pp. 1602-10, sep 1994.

[17] S. B. O'Sullivan, T. J. Schmitz, and G. D. Fulk, Physical rehabilitation.

[18] P. A. Ades, "Secondary Prevention of Coronary Heart Disease in Patients With Coronary," The New England Journal of Medicine, vol. 345, no. 12, pp. 892-902, 2004.

[19] P. A. Ades, M. L. Waldmann, W. J. McCann, and S. O. Weaver, "Predictors of cardiac rehabilitation participation in older coronary patients." Archives of internal medicine, vol. 152, no. 5, pp. 1033-5, may 1992.

[20] P. A. Ades, M. L. Waldmann, and C. Gillespie, "A controlled trial of exercise training in older coronary patients." The journals of gerontology. Series A, Biological sciences and medical sciences, vol. 50A, no. 1, pp. M7-11, jan 1995.

[21] W. S. Conn, S. G. Taylor, and P. Wiman, "Anxiety, Depression, Quality of Life, and Self-Care Among Survivors of Myocardial Infarction," Issues in Mental Health Nursing, vol. 12, no. 4, pp. 321-331, jan 1991.

[22] "NAO Evolution — Aldebaran." 